\title{
Parkinson's Disease - An Unusual Case of Prolonged Invasive Home Ventilation
}

\author{
Abdul Qayoom Dar, M.D., Murtaza Asif Ali, M.D. \\ Department of Anaesthesiology and Critical Care, SKIMS, Soura, Srinagar
}

A B S T R A C T

Parkinson's disease is a neurodegenerative disorder characterized by muscle rigidity, tremor, slowing or loss of physical movement. The impact of the disease is indicated by the fact that mortality is two to five times as high among affected persons as among age matched controls resulting in reduction in life expectancy. Amongst the motor dysfunction, swallowing disturbances can lead to drooling and impaired ability to swallow. These could cause aspiration pneumonia and death. We report a case of prolonged invasive home ventilation. Patient needs four hours of ventilation a day and uses Passy Muir Valve PMV 2000 for speech. JMS 2013;16(2):97-98

Keywords: Invasive Home Ventilation, Parkinson's disease, Tracheotomy

\section{INTRODUCTION}

Parkinson's disease is a neurodegenerative disorder that impairs the sufferer's motor skills and speech. ${ }^{1}$ Parkinson's disease (PD) named after English physician James Parkinson, is characterized by tremor, rigidity, bradykinesia or akinesia. Age is the single most consistent risk factor. Mortality is two to five times higher among affected persons as compared to age matched controls. ${ }^{2}$ Motor dysfunction with impaired swallowing can cause aspiration pneumonia and death. We present a case of prolonged invasive home ventilation in a patient of $\mathrm{PD}$ for more than 18 months. In absence of a favorable outcome, this has huge financial and social implications.

\section{CASE REPORT}

60 years old woman with Parkinson's disease for last 15 years was admitted in July 2007 with complaints of breathlessness and fever with chills for three days. She was being treated with a combination of levodopa, carbidopa and ropinirole. On examination, she was conscious, pulse rate was $100 / \mathrm{min}$ and blood pressure was $120 / 70 \mathrm{mmHg}$.

\section{Correspondence}

Dr. Abdul Qayoom Dar, M.D.

Additional Professor

Department of Anaesthesiology and Critical Care

Sher-i-Kashmir Institute of Medical Sciences, Soura, Srinagar Email: qayoom777@gmail.com
She had shallow breathing, few crepitations were present at lung bases. On neurological examination she had bradykinesia and increased muscle tone. Cranial nerve function was intact. Sensory and cerebellar functions were intact. Chest X Ray revealed haziness in both bases. Arterial Blood Gas analysis revealed type II respiratory failure. A diagnosis of Parkinson's disease with sepsis due to aspiration pneumonia was made.

Patient's Glasgow Coma Scale Score dropped to 3/15. Patient was shifted to the Intensive Care Unit (ICU), ventilated with Synchronized Intermittent Mandatory Ventilation mode, and sedated with midazolam and morphine. Antibiotics (ceftriaxone, azithromycin, moxifloxacin) were started. Nasogastric (NG) tube, an indwelling arterial and a right internal jugular venous triple lumen catheter were put in. NG tube feeding, antacid prophylaxis and thrombo - prophylaxis was started. Baseline investigations were unremarkable, and Thyroid Function Tests were normal.

Tracheostomy was performed under general anesthesia. Patient was weaned off to Pressure Support Ventilation (PSV) and Continuous Positive Airway Pressure (CPAP) mode. PS was gradually reduced and oxygen enriched air was given. She would need PSV and CPAP for $2-4$ hours during night. NG tube, central line and urinary catheter were removed. Patient was given normal diet, was ambulatory 
with support and maintained oxyhemoglobin saturation of $92 \%$ on room air. She had no cough reflex. Patient could speak using Passy Muir speaking valve. The relatives bought a ventilator and other necessary equipment to look after her at home. She was shifted home after approximately 150 days in ICU. January 2009, the patient continues to be on invasive home ventilation. She needs four hours of assistance during the night. She is brought to the ICU once a month for changing the tracheostomy tube.

\section{DISCUSSION}

Amongst the motor dysfunction in PD, impaired ability to swallow can cause choking and aspiration pneumonia that can lead to death. Ventilation in PD is impaired due to reduction in maximum voluntary ventilation, mid expiratory flow rate and forced expiratory volume in one second $^{3}$. Impairment in ventilation was reported in $87 \%$ patients with $\mathrm{PD}^{4}$. Airway obstruction may be present in $\mathrm{PD}$ due to increased parasympathetic tone ${ }^{5}$. The defect in ventilation in PD appears to be due to rigidity and weakness of respiratory muscles ${ }^{6}$. The results of a prospective study indicated that the central neural mechanism sub serving the recruitment of motor units during voluntary and reflex cough are impaired in patients with $\mathrm{PD}^{7}$. Impairment in the control of the epiglottic, laryngeal, and pharyngeal musculature lead to disordered swallowing ${ }^{8}$, making these patients silent aspirators and in combination with defective motor control of coughing, highly susceptible to aspiration pneumonia. This has been described as the most common cause of death in $\mathrm{PD}^{9}$.

Our patient presented to the hospital with respiratory failure secondary to aspiration pneumonia. Although she was resuscitated and ventilated in ICU, she never could cough out secretions from the respiratory tract. This led to unsuccessful attempts to wean her off the mechanical ventilation. She occupied a bed in ICU for five months leading at times to cancellation of routine cardiac and neurosurgical procedures. The relatives came to our rescue who arranged equipment and manpower support to shift her home. Invasive home ventilation without a favorable outcome has huge financial and social implications, causing a drain on resources of the family as well as psycho-social problems in looking after such a patient at home. We request the readers to comment on the pros and cons of this case and critically comment on our decision to ventilate such a patient. Since euthanasia is not practiced in most countries in the world, would denial of respiratory support to such a patient be accepted or after prior counseling of the relatives, should "Do Not Resuscitate" orders have been in place?

\section{REFERENCES}

1. Jankovic J. "Parkinson's disease: clinical features and diagnosis". J Neurol Neurosurg Psychiatr 2008;79(4): 368-76.

2. Lang AE and Lozano AM. "Parkinson's Disease Review Article". The New England Journal of Medicine 1998;339:1044-53.

3. Lilker ES, and Woolf CR. Pulmonary Function in Parkinson's syndrome. Canadian Medical Journal 1968; 99:752 -57.

4. Obenour WH, Stevens PM, Cohen AA and McCutchen JJ. The causes of abnormal pulmonary function in Parkinson's disease. American Review of Respiratory Diseases 1972;105:382-87.

5. Neu HC, Connolly JJ, Schwertey FW, Ladwig HA and Brody AW. Obstructive respiratory dysfunction in Parkinsonian patients. American Review of Respiratory Diseases 1967;95: 33-47.

6. Mehta AD, Wright WB and Kirby JB. Ventilatory function in Parkinson's disease. British Medical Journal 1978;1456-57.

7 . Giovanni AF, Pantaleo T, Lavorini F, Benvenuti F and Gangemi S. Defective Motor Control of Coughing in Parkinson's disease. American Journal of Critical Care Medicine 1998;158:458-64.

8. Robbins J A, Logemann JA and Kirshner HS. Swallowing and speech production in Parkinson's disease. Ann Neurol 1986;19:283 - 87.

9. Hoehn $M$ and Yahr M D. Parkinsonism: Onset, progression and mortality. Neurology 1967;34: 868 -76. 